\title{
The Value of Prostate-Specific Antigen-Related Indexes and Imaging Screening in the Diagnosis of Prostate Cancer
}

This article was published in the following Dove Press journal: Cancer Management and Research

\author{
Xiaojing Bai ${ }^{1,2, *}$ \\ Yumei Jiang ${ }^{1,2, *}$ \\ Xinwei Zhang ${ }^{1,2}$ \\ Meiyu Wang ${ }^{3}$ \\ Juanhua Tian' \\ Lijun $\mathrm{Mu}^{\prime}$ \\ Yuefeng $\mathrm{Du}{ }^{1,2}$
}

'Department of Urology, The First Affiliated Hospital of Xi'an Jiaotong University, Xi'an, Shaanxi 71006I, People's Republic of China; ${ }^{2}$ Oncology Research Lab, Key Laboratory of Environment and Genes Related to Diseases, Ministry of Education, Xi'an, Shaanxi 710061, People's Republic of China; ${ }^{3}$ Department of Imaging, The First Affiliated Hospital of Xi'an Jiaotong University, Xi'an, Shaanxi 71006I,

People's Republic of China

*These authors contributed equally to this work
Correspondence: Yuefeng Du Department of Urology, The First Affiliated Hospital of Xi'an Jiaotong University, 277 Western Yanta Road, Xi'an, Shaanxi 71006I, People's Republic of China

Tel/Fax +86-29-8532-3945

Email duyuefeng_123@I63.com
Objective: The aim of this study was to explore the value of the prostate-specific antigen (PSA) levels, the ratio of free PSA to total PSA (fPSA/TPSA), the PSA density (PSAD), digital rectal examination (DRE), transrectal prostate ultrasound (TRUS), and multiparameter MRI (MP-MRI) in the differential diagnosis of benign prostatic hyperplasia (BPH) and prostate cancer $(\mathrm{PCa})$.

Methods: From February 2016 to September 2019, data from 620 patients who underwent systematic transrectal ultrasound-guided prostate biopsy (STURS-PB) in our hospital were retrospectively collected, including the PSA levels, the fPSA/TPSA ratio, the PSAD, DRE, TRUS, MP-MRI, prostate volume, and other clinical data.

Results: Among the 620 patients, 249 patients were in the PCa group, and 371 patients in the BPH group. The positive puncture rate was $40.16 \%$. The positive predictive values of DRE, TRUS, mpMRI, and TPSA levels for PCa were 39.91\%, 39.38\%, 64.14\%, and $41.57 \%$, respectively; the sensitivity of these parameters was $37.35 \%, 51.41 \%, 74.69 \%$, and $57.43 \%$, respectively; and the specificity of these parameters was $62.26 \%, 46.90 \%$, $71.97 \%$, and $45.82 \%$, respectively. When the TPSA concentration was in the range of 4-20 ng/mL, the positive puncture rate of STURS-PB was $23.18 \%$, with a high rate of misdiagnosis. When the TPSA concentration was in the range of 4-20 ng/mL, the fPSA/ TPSA ratio was 0.15 , the PSAD was 0.16 , the comprehensive evaluation of PCa was optimal (the sensitivity of these parameters was $88.85 \%$ and $84.09 \%$, respectively; the specificity was $80.17 \%$ and $67.29 \%$, respectively; the positive predictive value was $57.41 \%$ and $51.39 \%$, respectively). When the TPSA concentration $>4 \mathrm{ng} / \mathrm{mL}$, the fPSA/TPSA ratio $\leq 0.15$ and the PSAD $\geq 0.16$, the sensitivity, specificity, and correctness index of the PCa and BPH diagnosis were $80.54 \%, 82.75 \%$, and $67.07 \%$, respectively.

Conclusion: When using DRE, TRUS, and MP-MRI to screen for PCa, MP-MRI has a relatively high sensitivity and specificity. Using these three thresholds (TPSA $>4 \mathrm{ng} / \mathrm{mL}$ combined with an PPSA/TPSA ratio $\leq 0.15$ and a PSAD $\geq 0.16$ ) is significantly better than using TPSA levels alone for the differential diagnosis of $\mathrm{PCa}$ and $\mathrm{BPH}$.

Keywords: total prostate-specific antigen, free prostate-specific antigen, prostate-specific antigen density, prostate cancer, benign prostatic hyperplasia

\section{Introduction}

Prostate cancer (PCa) is the most common male tumor in Europe and the US, and it has the second highest mortality rate among male tumors. Although the incidence of prostate cancer in Asia is lower than that in Europe and the US, it has been increasing rapidly in recent years. ${ }^{1,2}$ It has been reported that the 5-year survival rate of early PCa 
can be as high as $99 \%$ after timely and reasonable treatment. Therefore, diagnosing PCa safely, early, and accurately has been a major focus of urologists. ${ }^{3-5}$

At present, the diagnostic methods of $\mathrm{PCa}$ include digital rectal examination (DRE), prostate-specific antigen (PSA), transrectal prostate ultrasound (TRUS), and multiparameter MRI (MP-MRI), but systematic transrectal ultrasound-guided prostate biopsy (STURS-PB) is the gold standard for the diagnosis of PCa. ${ }^{1,4}$ STURS-PB can obtain prostate tissue samples and make pathological diagnoses of PCa. ${ }^{6-9}$ However, because STURS-PB is an invasive test, the procedure is painful and difficult for patients, and it can be accompanied by many complications, such as hematuria, urinary retention, hemospermia, and infection, which are not suitable for routine screening. ${ }^{6-9}$ Therefore, it is of great clinical significance to determine the need for STURS-PB according to the results of DRE, PSA, PSA-related indexes, TRUS, and MP-MRI. Although DRE, PSA, PSA-related indexes, TRUS, and MP-MRI can significantly improve the positive diagnostic rate of STURS-PB and reduce unnecessary punctures and economic burdens for patients, there are also problems with these methods, such as insufficient sensitivity and specificity, especially when the PSA ranges from $4-20 \mathrm{ng} / \mathrm{mL} .{ }^{10-15}$ Accordingly, the clinical data from 620 patients who underwent STURS-PB in our hospital were collected, and the patients were divided into two groups (BPH group and PCa group). The diagnostic efficiency of TRUS, DRE, and MP-MRI was analyzed, and the critical values of the PPSA/TPSA ratio and the PSAD for diagnosing PCa was determined when the TPSA levels ranged from 4-20 ng/mL. Furthermore, the clinical value of the fPSA/TPSA and the PSAD thresholds for the differential diagnosis of PCa and BPH when the TPSA levels $>4$ $\mathrm{ng} / \mathrm{mL}$ was discussed.

\section{Data and Methods}

\section{Inclusion Criteria and Exclusion Criteria}

The clinical data of 620 patients who underwent STURSPB in our hospital from February 2016 to September 2019 were collected and analyzed retrospectively. The inclusion criteria were as follows: 1) STURS-PB patients; 2) pathological diagnosis of PCa or BPH; and 3) complete clinical data. The exclusion criteria were as follows: 1) pathological diagnosis was not prostate cancer or hyperplasia; and 2) incomplete clinical data. The study was performed in accordance with the ethical standards of the institutional and/or national research committee and with the 1964 Declaration of Helsinki and its later amendments or comparable ethical standards. The study was approved by the ethics committee of The First Affiliated Hospital of Xi'an Jiaotong University and all participants signed informed consent.

\section{STURS-PB and Clinical Data Collection}

STURS-PB has been described in previous research. ${ }^{16,17}$ The clinical data, including the PSA levels1, the fPSA levels, the prostate volume, the PPSA/TPSA ratio, the PSAD, transrectal ultrasound, DRE and MP-MRI, were collected and analyzed. The TPSA and fPSA levels are determined by the chemiluminescence method. The detection instrument was an automatic chemiluminescence immunoanalyzer from Beckman Coulter Company in the US. The kit was provided by the company and operated by special personnel. The ultrasound diagnosis was performed using the PHILIPS HD-11 GE-VOLUSION 730 EXPERT color Doppler ultrasound diagnostic instrument, and the probe frequency was $6-12 \mathrm{MHz}$. The prostate was observed by the rectum; the anterior, posterior, left, right, upper diameter, and lower diameter of the prostate were measured. The prostate volume was calculated according to the formula: prostate volume $=$ (front and rear diameter $\times$ left and right diameter $\times$ upper and lower diameter $) \times \pi / 6$. The PSAD was calculated according to the following formula: $\mathrm{PSAD}=\mathrm{PSA} /$ prostate volume. MP-MRI was performed using a ge-3.0 clinical MRI scanner (twin speed scanner, General Electric Medical System, USA).

\section{Statistical Methods}

SPSS19.0 statistical software was used for analysis, and the measurement data were expressed as $\mathrm{x} \pm \mathrm{s}$. The onesample K-S test was used to assess the normal distribution of continuous variables before performing further comparisons. Student's $t$-test and the Wilcoxon test were used to compare clinical characteristics between the $\mathrm{PCa}$ group and $\mathrm{BPH}$ group. Categorical data were expressed as percentages, and comparisons between groups were made using the $\chi^{2}$ test or Fisher's exact test. When $P<0.05$, the difference was significant.

\section{Results}

Positive Predictive Value, Sensitivity, and Specificity Analysis of DRE, TRUS, MP-MRI, and TPSA Levels

Among 620 patients, 249 patients were in the PCa group, and 371 patients were in the BPH group. The positive 
puncture rate was $40.16 \%$. As shown in Table 1 , the positive predictive values of DRE, TRUS, mpMRI, and the TPSA levels for PCa were $39.91 \%, 39.38 \%, 64.14 \%$, and $41.57 \%$, respectively; the sensitivity of these parameters was $37.35 \%$, $51.41 \%, 74.69 \%$, and $57.43 \%$, respectively; and the specificity of these parameter was $62.26 \%, 46.90 \%, 71.97 \%$, and $45.82 \%$, respectively. As shown in Table 2, when the TPSA levels ranged from 4-10 $\mathrm{ng} / \mathrm{mL}$ and $10-20 \mathrm{ng} / \mathrm{mL}$, the positive puncture rates for STURS-PB are $17.24 \%$ and $25.97 \%$, respectively. When the TPSA levels ranged from 4-20 ng/mL, the positive puncture rate for STURS-PB was $23.18 \%$. The results showed that when the PSA ranged from 4-20 ng/mL, the positive puncture rate for STURS-PB was not high, and there was a high negative puncture rate.

\section{Comparison of the TPSA Levels, fPSA/} TPSA Ratio, Prostate Volume, and PSAD Between the BPH Group and PCa Group When the TPSA Levels Were in the

\section{Range of 4-20 ng/mL}

As shown in Table 3, there was no significant difference in the TPSA levels ( $11.02 \pm 5.14$ vs $12.25 \pm 4.52, P=0.109)$ between the $\mathrm{BPH}$ group and the $\mathrm{PCa}$ group. The fPSA/TPSA ratio $(0.23 \pm 0.17$ vs $0.15 \pm 0.06, P=0.005)$ and prostate volume (71.79 \pm 32.45 vs $41.51 \pm 19.12, P=0.021)$ were significantly higher in the $\mathrm{BPH}$ group than in the $\mathrm{PCa}$ group, but the

Table I Analysis of the Positive Predictive Value, Sensitivity, and Specificity of Different Examination Methods

\begin{tabular}{|l|l|l|l|}
\hline Parameter & $\begin{array}{l}\text { Positive } \\
\text { Predictive } \\
\text { Value }\end{array}$ & Sensitivity & Specificity \\
\hline DRE & $39.91 \%(93 / 233)$ & $37.35 \%(93 / 249)$ & $62.26 \%(23 \mathrm{I} / 37 \mathrm{I})$ \\
TRUS & $39.38 \%(128 / 325)$ & $51.41 \%(128 / 249)$ & $46.90 \%(174 / 37 \mathrm{I})$ \\
$\mathrm{mPMRI}$ & $64.14 \%(186 / 290)$ & $74.69 \%(186 / 249)$ & $71.97 \%(267 / 37 \mathrm{I})$ \\
TPSA (ng/mL) & $41.57 \%(143 / 344)$ & $57.43 \%(143 / 249)$ & $45.82 \%(170 / 37 \mathrm{I})$ \\
\hline
\end{tabular}

Abbreviations: DRE, digital rectal examination; TPSA, total prostate-specific antigen; TRUS, transrectal prostate ultrasound; mPMRI, multiparameter MRI.

Table 2 Comparison of the Puncture Positive Rate Across Different TPSA Concentrations

\begin{tabular}{|l|l|l|}
\hline Parameter & Scope $(\mathbf{n})$ & Positive Puncture Rate $(\%, \mathbf{n})$ \\
\hline TPSA $(\mathrm{ng} / \mathrm{mL})$ & $4 \sim 10(145)$ & $17.24 \%(25 / 145)$ \\
& $10 \sim 20(308)$ & $25.97 \%(80 / 308)$ \\
& $4 \sim 20(453)$ & $23.18 \%(105 / 453)$ \\
& $>20(167)$ & $86.82 \%(144 / 167)$ \\
\hline
\end{tabular}

Table 3 Comparison of TPSA Levels, fPSA/TPSA Ratios, Prostate Volumes, and PSAD Levels Between the BPH Group and PCa Group When the TPSA Concentration Ranged from 4-20 ng/mL

\begin{tabular}{|l|l|l|l|l|}
\hline Group & $\begin{array}{l}\text { TPSA } \\
(\mathbf{n g} / \mathbf{m L})\end{array}$ & $\begin{array}{l}\text { FPSA/ } \\
\text { TPSA }\end{array}$ & $\begin{array}{l}\text { Prostate } \\
\text { Volume }(\mathbf{m L})\end{array}$ & PSAD \\
\hline BPH (348) & $11.02 \pm 5.14$ & $0.23 \pm 0.17$ & $71.79 \pm 32.45$ & $0.13 \pm 0.06$ \\
PCa (105) & $12.25 \pm 4.52$ & $0.15 \pm 0.06$ & $41.51 \pm 19.12$ & $0.21 \pm 0.11$ \\
$P$ & 0.109 & 0.005 & 0.021 & 0.011 \\
\hline
\end{tabular}

PSAD $(71.79 \pm 32.45$ vs $41.51 \pm 19.12, P=0.011)$ was significantly lower in the BPH group than in the PCa group $(P<0.01)$.

\section{When the TPSA Levels Ranged from 4-20 ng/mL, Comparison of Different fPSA/TPSA Thresholds in the Diagnosis of $\mathrm{PCa}$}

Table 4 shows the results of different fPSA/TPSA cutoff values for the sensitivity, specificity, and positive predictive values for BPH and PCa discrimination when the TPSA levels ranged from $4-20 \mathrm{ng} / \mathrm{mL}$. When the fPSA/TPSA ratio was 0.15 , the comprehensive evaluation of $\mathrm{PCa}$ screening was optimal (the sensitivity was $88.85 \%$, the specificity was $80.17 \%$, and the positive predictive value was $57.41 \%$ ).

\section{When the TPSA Levels Ranged from} 4-20 ng/mL, Comparison of Different PSAD Thresholds in the Diagnosis of PCa

Table 5 shows the results of different PSAD cutoff values for the sensitivity, specificity, and positive predictive values for $\mathrm{BPH}$ and PCa discrimination when the TPSA levels ranged from 4-20 ng/mL. When the PSAD was 0.16, the comprehensive evaluation of PCa screening was optimal (the sensitivity was $84.09 \%$, the specificity was $67.29 \%$, and the positive predictive value was $51.39 \%$ ).

\section{Comparison of Different Index} Thresholds for Diagnosing PCa When the TPSA Levels $>4 \mathrm{ng} / \mathrm{mL}$

Table 6 shows the sensitivity, specificity, and positive predictive values for $\mathrm{PCa}$ when the TPSA levels were $>4.0 \mathrm{ng} / \mathrm{mL}$, the PPSA/TPSA ratio $\leq 0.15$, and the PSAD $\geq 0.16$. As shown in Table 6 , when the TPSA levels were $>4.0 \mathrm{ng} / \mathrm{mL}$, the PPSA/TPSA ratio $\leq 0.15$, and the PSAD $\geq 0.16$, the comprehensive evaluation of PCa screening was optimal (the sensitivity was $80.54 \%$, the specificity was $82.75 \%$, and the positive predictive value was $67.07 \%$ ). 
Table 4 Comparison of Different fPSA/TPSA Thresholds to Diagnose PCa Between the BPH Group and the PCa Group When the TPSA Concentration Ranged from 4-20 ng/mL

\begin{tabular}{|l|l|l|l|l|l|l|l|}
\hline $\begin{array}{l}\text { fPSA/ } \\
\text { TPSA }\end{array}$ & $\begin{array}{l}\text { True } \\
\text { Positive }\end{array}$ & $\begin{array}{l}\text { False } \\
\text { Negative }\end{array}$ & $\begin{array}{l}\text { True } \\
\text { Negative }\end{array}$ & $\begin{array}{l}\text { False } \\
\text { Positive }\end{array}$ & $\begin{array}{l}\text { Sensitivity } \\
\text { (\%) }\end{array}$ & $\begin{array}{l}\text { Specificity } \\
\text { (\%) }\end{array}$ & $\begin{array}{l}\text { Positive Predictive } \\
\text { Value (\%) }\end{array}$ \\
\hline 0.13 & 66 & 39 & 315 & 33 & 62.86 & 90.51 & 66.67 \\
0.14 & 84 & 21 & 291 & 57 & 80.00 & 83.26 & 59.57 \\
0.15 & 93 & 12 & 279 & 69 & 88.85 & 80.17 & 57.41 \\
0.16 & 96 & 9 & 258 & 99 & 91.43 & 74.14 & 49.23 \\
0.17 & 99 & 6 & 216 & 132 & 94.28 & 62.07 & 42.86 \\
\hline
\end{tabular}

Table 5 Comparison of Different PSAD Thresholds to Diagnose PCa Between the BPH Group and the PCa Group When the TPSA Concentration Ranged from 4-20 ng/mL

\begin{tabular}{|l|l|l|l|l|l|l|l|}
\hline PSAD & $\begin{array}{l}\text { True } \\
\text { Positive }\end{array}$ & $\begin{array}{l}\text { False } \\
\text { Negative }\end{array}$ & $\begin{array}{l}\text { True } \\
\text { Negative }\end{array}$ & $\begin{array}{l}\text { False } \\
\text { Positive }\end{array}$ & $\begin{array}{l}\text { Sensitivity } \\
\text { (\%) }\end{array}$ & $\begin{array}{l}\text { Specificity } \\
\text { (\%) }\end{array}$ & $\begin{array}{l}\text { Positive Predictive } \\
\text { Value (\%) }\end{array}$ \\
\hline 0.14 & 123 & 9 & 66 & 255 & 93.18 & 20.56 & 32.29 \\
0.15 & 114 & 18 & 135 & 186 & 86.36 & 42.06 & 38.00 \\
0.16 & 111 & 21 & 216 & 105 & 84.09 & 67.29 & 51.39 \\
0.17 & 99 & 33 & 240 & 81 & 75.00 & 74.76 & 55.00 \\
0.18 & 84 & 48 & 273 & 48 & 63.63 & 85.05 & 63.63 \\
\hline
\end{tabular}

Table 6 Comparison of Diagnostic Values of PCa When the TPSA Concentration $>4 \mathrm{ng} / \mathrm{mL}$

\begin{tabular}{|l|l|l|l|}
\hline $\begin{array}{l}\text { Diagnostic } \\
\text { Value }\end{array}$ & $\begin{array}{l}\text { FPSA } / \\
\text { TPSA } \leq \mathbf{0 . 1 5}\end{array}$ & PSAD $\geq \mathbf{0 . 1 6}$ & $\begin{array}{l}\text { FPSA/TPSA } \leq \mathbf{0 . 1 5} \\
\text { PSAD } \geq \mathbf{0 . 1 6}\end{array}$ \\
\hline $\begin{array}{l}\text { True positive } \\
\text { True negative }\end{array}$ & 179 & 169 & 149 \\
False positive & 195 & 300 & 360 \\
False negative & 30 & 114 & 75 \\
\hline Total & 620 & 620 & 36 \\
\hline Sensitivity (\%) & 85.64 & 82.04 & 80.54 \\
Specificity (\%) & 52.55 & 72.46 & 82.75 \\
Correct index & 38.19 & 48.18 & 67.07 \\
\hline
\end{tabular}

\section{Discussion}

$\mathrm{PCa}$ is one of the most common malignant diseases in males, and the incidence rate has been increasing in recent years. ${ }^{1,3}$ The early detection and diagnosis of PCa is of great clinical significance. ${ }^{1,3}$ DRE and TRUS are important methods for the early detection of PCa. They have the advantages of simplicity and reproducibility, but both have the disadvantages of low specificity and sensitivity. ${ }^{18-20}$ It has been reported that the false negative rate of TRUS is as high as $30-45 \%$, while the missed diagnosis rate of DRE alone is as high as $48-85 \% .{ }^{21-23}$ In this study, we found that the positive predictive value of DRE and TRUS in PCa screening was $39.91 \%$ and $39.38 \%$, respectively; the sensitivity was $37.35 \%$ and $51.41 \%$, respectively; and the specificity was $62.26 \%$ and $46.90 \%$, respectively. The results suggested that the sensitivity and specificity of TRUS for PCa were low, but the sensitivity and specificity of DRE for PCa were relatively high. The reasons for this result are mainly related to the size of the lesion, the location of the lesion, the level of operator experience, and the subjective consciousness of the examiner. In recent years, MP-MRI has attracted increasing attention in the early diagnosis of PCa. It has been reported that the sensitivity and specificity of MP-MRI for PCa diagnosis range from $54-82 \%$ and $46-91 \%$, respectively. ${ }^{24,25}$ In this study, we found that the positive predictive value of MP-MRI for PCa diagnosis was $64.14 \%$, the sensitivity was $74.69 \%$, and the specificity was $71.97 \%$. The results suggest that multiparameter MRI has high sensitivity and specificity in the diagnosis of $\mathrm{PCa}$ and has important clinical value for the early screening of PCa.

PSA is the most important marker for the early detection and identification of PCa. It has high organ specificity (not disease specificity), so both PCa and BPH lead to an increase in the PSA. ${ }^{26-28}$ It is suggested that there is a large overlap of PSA levels in BPH and PCa patients, so the examination of PSA levels alone affects the differential diagnosis of $\mathrm{BPH}$ and $\mathrm{PCa} .{ }^{26-28}$ In this study, we found that the positive predictive value of the PSA alone 
for the diagnosis of PCa was $41.57 \%$, the sensitivity was $57.43 \%$, and the specificity was $45.82 \%$. The results suggest that the PSA alone has low sensitivity and specificity in the diagnosis of PCa. Currently, a novel immunosensor constructed via the assembly of prostate-specific antibodies on the surface of Au@Pt NCs shows high sensitivity and specificity and shows promise. ${ }^{29,30}$ It is suggested that the serum PSA level is above the normal range in approximately $21-47 \%$ of BPH patients, and a high PSA level leads to unnecessary STURS-PB and limits its value as a screening method for $\mathrm{PCa} .{ }^{28,31,32}$ In this study, we found that when the TPSA levels ranged from 4-20 ng/mL, there was no significant difference in the TPSA concentration between the BPH group and the PCa group $(11.08 \pm 5.14 \mathrm{vs}$ $12.25 \pm 4.52, P=0.109$ ), and the positive puncture rate of STURS-PB was $23.18 \%$. The results showed that when the PSA ranged from 4-20 ng/mL, the TPSA levels of the $\mathrm{BPH}$ group and $\mathrm{PCa}$ group overlapped, the positive puncture rate of STURS-PB was not high, and the negative puncture rate was high.

At present, it is suggested that the fPSA/TPSA ratio and the PSAD can increase the positive predictive value of PSA for PCa when the PSA concentration ranges from 4-10 $\mathrm{ng} / \mathrm{mL}$, thus reducing unnecessary biopsies. ${ }^{33}$ Because of the overlap of TPSA levels between the BPH group and PCa group at a concentration of 4-20 ng/mL and because of the high negative puncture rate, we explored the optimal values for the PPSA/TPSA ratio and the PSAD for diagnosing PCa when the TPSA levels ranged from 4-20 ng/mL. In this study, we found that when the PSA levels ranged from 4-20 $\mathrm{ng} / \mathrm{mL}$, the fPSA/TPSA ratio and the PSAD were significantly different between the PCa group and BPH group, and when the fPSA/TPSA ratio was equal to or less than 0.15 and the PSAD was equal to or greater than 0.16 , the screening and diagnosis efficiency of PCa was optimal (the sensitivity of these parameters was $88.85 \%$ and $80.17 \%$, and the specificity was $84.09 \%$ and $67.29 \%$, respectively). The threshold of the PSAD $\geq 0.16$ is selected in this paper, as the sensitivity is higher for this threshold than the sensitivity obtained when using the recommended threshold of PSAD $>0.15$ to diagnose $\mathrm{PCa}(52.0-67.9 \%$ ); however, the specificity is still relatively low compared to $78.9-81.0 \%{ }^{32,34}$ The reason for this result may be related to the different research objects, reagents, and detection methods. Because there are some limitations when using PSA $>4 \mathrm{ng} / \mathrm{mL}$ as the threshold of PCa screening, ${ }^{34,35}$ we explored the diagnostic efficiency of using an fPSA/TPSA $\leq 0.15$ and a PSAD $\geq 0.16$ as thresholds for diagnosing PCa when the PSA levels were $>$
$4 \mathrm{ng} / \mathrm{mL}$. In this study, we used the principle of combining sensitivity and specificity to find that when using these three thresholds (PSA $>4 \mathrm{ng} / \mathrm{mL}$, fPSA/TPSA $\leq 0.15$ and PSAD $\geq 0.16$ ) to diagnose $\mathrm{PCa}$ and $\mathrm{BPH}$, the sensitivity, specificity, and positive predictive value were $80.54 \%, 82.75 \%$, and $67.07 \%$, respectively. Therefore, in the differential diagnosis of BPH and PCa, a TPSA level $>4 \mathrm{ng} / \mathrm{mL}$ combined with an $\mathrm{fPSA} / \mathrm{TPSA}$ ratio $\leq 0.15$ and a PSAD $\geq 0.16$ is recommended for optimal screening results. However, the following limitations still exist in this study: 1) this is a retrospective study with inherent limitations and bias risks; 2) the sample size of this study is small, and the research results need to be further validated across multiple centers and with a large sample size; and 3) the research results only represent the cases in our center and cannot be extended to different groups and units.

\section{Funding}

This study was supported by the National Natural Science Foundation of China (NSFC81372736) and the Project of Shaanxi Provincial Natural Science Basic Research Program (2020JM-370, 2020JM-390).

\section{Disclosure}

The authors report no conflicts of interest in this work.

\section{References}

1. Chang AJ, Autio KA, Scher HI. High-risk prostate cancer-classification and therapy. Nat Rev Clin Oncol. 2014;11(6):308-323. doi:10.1038/ nrclinonc. 2014.68

2. Center MM, Jemal A, Lortet-Tieulent J. International variation in prostate cancer incidence and mortality rates. Eur Urol. 2012;61 (6):1079-1092. doi:10.1016/j.eururo.2012.02.054

3. Litwin MS, Tan HJ. The diagnosis and treatment of prostate cancer: a review. JAMA. 2017;317(24):2532-2542. doi:10.1001/jama.2017.7248

4. Freedland S. Prostate cancer: race and prostate cancer personalized medicine: the future. Nat Rev Urol. 2018;15(4):207-208. doi:10.1038/ nrurol.2017.215

5. Hoogland AM, Kweldam CF, van Leenders GJ. Prognostic histopathological and molecular markers on prostate cancer needle-biopsies: a review. Biomed Res Int. 2014;2014:341324. doi:10.1155/2014/341324

6. Schoots IG, Roobol MJ, Nieboer D, et al. Magnetic resonance imaging-targeted biopsy may enhance the diagnostic accuracy of significant prostate cancer detection compared to standard transrectal ultrasound-guided biopsy: a systematic review and meta-analysis. Eur Urol. 2015;68(3):438-450.

7. Borghesi M, Ahmed H, Nam R, et al. Complications after systematic, random, and image-guided prostate biopsy. Eur Urol. 2017;71 (3):353-365. doi:10.1016/j.eururo.2016.08.004

8. Schouten MG, Barentsz JO, Thompson LC, et al. Why and where do we miss significant prostate cancer with multi-parametric magnetic resonance imaging followed by magnetic resonance-guided and transrectal ultrasound-guided biopsy in biopsy-naïve men? Eur Urol. 2017;71(6):896-903. doi:10.1016/j.eururo.2016.12.006 
9. Baco E, Rud E, Eri LM, et al. A randomized controlled trial to assess and compare the outcomes of two-core prostate biopsy guided by fused magnetic resonance and transrectal ultrasound images and traditional 12-core systematic biopsy. Eur Urol. 2016;69 (1):149-156. doi:10.1016/j.eururo.2015.03.041

10. Moul JW. Comparison of DRE and PSA in the detection of prostate cancer. J Urol. 2017;197(2S):S208-S209. doi:10.1016/j.juro.2016.11.031

11. Quaas J. PSA screening for prostate cancer. Am Fam Physician. 2015;91(9).

12. Thompson JE, Stricker PD. Diagnostic accuracy of multi-parametric MRI and transrectal ultrasound-guided biopsy in prostate cancer. Lancet. 2017;389(10071):767-768. doi:10.1016/S0140-6736(17)30121-6

13. Mayor S. Targeted biopsy with MRI and ultrasound improves detection of high risk prostate cancer. BMJ. 2015;350:h472.

14. Carlsson S, Vickers A, Lilja H, Hugosson J. Screening for prostate cancer. Ann Intern Med. 2012;156(7):539. doi:10.7326/0003-4819156-7-201204030-00014

15. Kim KS, Jeong WS, Park SY, et al. The effect of two weeks of treatment with dutasteride on bleeding after transurethral resection of the prostate. World J Mens Health. 2015;33(1):14-19. doi:10.5534/ wjmh.2015.33.1.14

16. Han M, Chang D, Kim C, et al. Geometric evaluation of systematic transrectal ultrasound guided prostate biopsy. J Urol. 2012;188 (6):2404-2409. doi:10.1016/j.juro.2012.07.107

17. Wu YP, Li XD, Ke ZB, et al. Risk factors for infectious complications following transrectal ultrasound-guided prostate biopsy. Infect Drug Resist. 2018;11:1491-1497. doi:10.2147/IDR.S171162

18. Carvalhal GF, Smith DS, Mager DE. Digital rectal examination for detecting prostate cancer at prostate specific antigen levels of $4 \mathrm{ng}$./ mL. or less. J Urol. 1999;161(3):835-839. doi:10.1016/S0022-53 47(01)61785-3

19. Catalona WJ, Richie JP, Ahmann FR, et al. Comparison of digital rectal examination and serum prostate specific antigen in the early detection of prostate cancer: results of a multicenter clinical trial of 6630 men. J Urol. 2017;197(2S):S200-S207. doi:10.1016/j. juro.2016.10.073

20. Catalona WJ, Partin AW, Finlay JA, et al. Use of percentage of free prostate-specific antigen to identify men at high risk of prostate cancer when PSA levels are 2.51 to $4 \mathrm{ng} / \mathrm{mL}$ and digital rectal examination is not suspicious for prostate cancer: an alternative model. Urology. 1999;54(2):220-224. doi:10.1016/S0090-4295(99) 00185-5

21. Mueller EJ, Crain TW, Thompson IM. An evaluation of serial digital rectal examinations in screening for prostate cancer. J Urol. 1988;140 (6):1445-1447. doi:10.1016/S0022-5347(17)42069-6

22. Halpern E, Strup S. Using gray-scale and color and power Doppler sonography to detect prostatic cancer. AJR Am J Roentgenol. 2000;174(3):623-627. doi:10.2214/ajr.174.3.1740623

23. El-Shater Bosaily A, Parker C, Brown LC. PROMIS-Prostate MR imaging study: a paired validating cohort study evaluating the role of multi-parametric MRI in men with clinical suspicion of prostate cancer. Contemp Clin Trials. 2015;42:26-40. doi:10.1016/j. cct. 2015.02 .008
24. Graser A, Heuck A, Sommer B, et al. Per-sextant localization and staging of prostate cancer: correlation imaging findings with whole-mount step section histopathology. AJR Am J Roentgenol. 2007;188(1):84-90. doi:10.2214/AJR.06.0401

25. Haider MA, Tanguay J, Evans AJ. Combined T2-weighted and diffusion-weighted MRI for localization of prostate cancer. AJR Am J Roentgenol. 2007;189(2):323-328. doi:10.2214/AJR.07.2211

26. Froehner M, Hakenberg OW, Koch R, et al. Comparison of the clinical value of complexed PSA and total PSA in the discrimination between benign prostatic hyperplasia and prostate cancer. Urol Int. 2006;76(1):27-30. doi:10.1159/000089731

27. Helfand BT, Anderson CB, Fought A, et al. Postoperative PSA and PSA velocity identify presence of prostate cancer after various surgical interventions for benign prostatic hyperplasia. Urology. 2009;74 (1):177-183. doi:10.1016/j.urology.2008.10.062

28. Sershon PD, Barry MJ, Oesterling JE. Serum prostate-specific antigen discriminates weakly between men with benign prostatic hyperplasia and patients with organ-confined prostate cancer. Eur Urol. 1994;25(4):281-287. doi:10.1159/000475302

29. Wang R, Liu W, Wang A, Xue Y, Wu L, Feng J. A new label-free electrochemical immunosensor based on dendritic Core-Shell AuPd@Au nanocrystals for highly sensitive detection of prostate specific antigen. Biosens Bioelectron. 2018;99:458-463. doi:10.1016/j. bios.2017.08.010

30. Wang R, Wang A, Liu W, et al. A novel label-free electrochemical immunosensor for ultra-sensitively detecting prostate specific antigen based on the enhanced catalytic currents of oxygen reduction catalyzed by Core-Shell Au@Pt nanocrystals. Biosens Bioelectron. 2018;102:276-281. doi:10.1016/j.bios.2017.11.041

31. Hochberg DA, Armenakas NA, Fracchia JA. Relationship of prostate-specific antigen and prostate volume in patients with biopsy proven benign prostatic hyperplasia. Prostate. 2000;45(4):315-319. doi:10.1002/1097-0045(20001201)45:4<315::AID-PROS5>3.0. $\mathrm{CO} ; 2-2$

32. Fu S, Zhang X, Niu Y, Wang RT. Prostate specific antigen, mean platelet volume, and platelet distribution width in combination to discriminate prostate cancer from benign prostate hyperplasia. Asian Pac J Cancer Prev. 2018;19(3):699-702. doi:10.22034/APJCP.2018.19.3.699

33. Kang SH, Bae JH, Park HS, et al. Prostate-specific antigen adjusted for the transition zone volume as a second screening test: a prospective study of 248 cases. Int $J$ Urol. 2006;13:910-914. doi:10.1111/j.1442-2042.2006.01439.x

34. Seaman E, Whang M, Olsson CA, et al. PSA density (PSAD). Role in patient evaluation and management. Urol Clin North Am. 1993;20 (4):653-663.

35. Verma A, St Onge J, Dhillon K, Chorneyko A. PSA density improves prediction of prostate cancer. Can J Urol. 2014;21(3):7312-7321.

\section{Publish your work in this journal}

Cancer Management and Research is an international, peer-reviewed open access journal focusing on cancer research and the optimal use of preventative and integrated treatment interventions to achieve improved outcomes, enhanced survival and quality of life for the cancer patient.
The manuscript management system is completely online and includes a very quick and fair peer-review system, which is all easy to use. Visit http://www.dovepress.com/testimonials.php to read real quotes from published authors. 